\title{
Impact of 10-valent pneumococcal conjugate vaccine on invasive pneumococcal disease and
} nasopharyngeal carriage in Kenya

Laura L. Hammitt MD ${ }^{1,2,3}$, Anthony O. Etyang MB ChB ${ }^{1,4}$, Susan C. Morpeth FRACP ${ }^{1,2,4}$, John Ojal $\mathrm{PhD}^{1,4}$, Alex Mutuku MSc ${ }^{1}$, Neema Mturi MRCPCH ${ }^{1}$, Jennifer C. Moisi PhD ${ }^{1,2,5}$, Ifedayo M. Adetifa $\mathrm{PhD}^{1,4}$, Angela Karani $\mathrm{BSc}^{1}$, Donald O. Akech $\mathrm{BSc}^{1}$, Mark Otiende $\mathrm{MSc}^{1}$, Tahreni Bwanaali MBA ${ }^{1,2}$, Jackline Wafula BSN ${ }^{1}$, Christine Mataza $\mathrm{KRCHN}^{6}$, Edward Mumbo BSc ${ }^{6}$, Collins Tabu $\mathrm{MPH}^{7}$, Maria Deloria Knoll PhD ${ }^{3}$, Evasius Bauni PhD ${ }^{1}$, Kevin Marsh FMedSci ${ }^{1,2}$, Thomas N. Williams FMedSci ${ }^{1,8,9}$, Tatu Kamau MPH${ }^{7}$, Shahnaaz K. Sharif $\mathrm{MD}^{7}$, Orin S. Levine $\mathrm{PhD}^{3,10}$, J. Anthony G. Scott FRCP ${ }^{1,2,4,9}$

1. Epidemiology and Demography Department, KEMRI-Wellcome Trust Research Programme, Centre for Geographic Medicine-Coast, Kilifi, Kenya

2. Nuffield Department of Clinical Medicine, University of Oxford, Oxford, UK

3. Department of International Health, Johns Hopkins Bloomberg School of Public Health, Baltimore, Maryland, USA

4. Department of Infectious Disease Epidemiology, London School of Hygiene \& Tropical Medicine, London, UK

5. Pfizer Vaccines, Paris, France

6. County Health Department, Kilifi County, Kenya

7. National Vaccines and Immunization Programme, Ministry of Health, Kenya

8. Imperial College, London, UK

9. INDEPTH Network, Accra, Ghana

10. Bill and Melinda Gates Foundation, Seattle, USA

Word count: 3379

Key words: Streptococcus pneumoniae; pneumococcal conjugate vaccine; PHiD-CV; invasive pneumococcal disease; Kenya; vaccine effectiveness; nasopharyngeal carriage; catch up campaign

Corresponding author:

Laura Hammitt

415 N. Washington St., $4^{\text {th }}$ Floor

Baltimore, MD 21231

Tel: 443-651-0000

LHammitt@jhu.edu
Alternate corresponding author:

Anthony Scott

PO Box 230

Kilifi, Kenya 80108

Tel +254 417522063

Ascott@ikilifi.org 


\section{Abstract.}

Background: 10-valent pneumococcal conjugate vaccine (PCV10), delivered at 6, 10 and 14 weeks of age, was introduced in Kenya in January 2011, accompanied by a catch-up campaign in Kilifi County for children $<5$ years. Coverage with $\geq 2$ PCV10 doses in children 2-11 months was 80\% in 2011 and $84 \%$ in 2016; coverage with $\geq 1$ dose in children $12-59$ months was $66 \%$ and $87 \%$, respectively.

Methods: Clinical and microbiological surveillance for invasive pneumococcal disease (IPD) among admissions of all ages at Kilifi County Hospital was linked to the Kilifi Health and Demographic Surveillance System from 1999-2016. We calculated the incidence rate ratio (IRR) comparing the pre-vaccine and postvaccine eras, adjusted for confounding, and reported percent reduction in IPD as 1-IRR. Annual crosssectional surveys of nasopharyngeal carriage were conducted from 2009-2016.

Findings: Surveillance identified 667 IPD cases in 3,211,403 person-years of observation. IPD incidence in children < 5 years fell sharply in 2011 following PCV10 introduction, and remained low (PCV10-type IPD: 60·8 vs 3.2/100,000 [92\% reduction; $95 \% \mathrm{Cl}: 78,97$ ]; overall IPD: $81 \cdot 6$ vs $15 \cdot 3 / 100,000$ [68\% reduction; $95 \% \mathrm{Cl}: 40$, 83]; 1999-2010 vs 2012-2016). PCV10-type IPD also declined significantly in unvaccinated age groups (<2 months, $5-14$ years, $\geq 15$ years), with estimated reductions of $100 \%, 74 \%$, and $81 \%$, respectively. There was no significant change in the incidence of non-PCV10 type IPD. In children aged $<5$ years, PCV10-type carriage declined by $74 \%$ and non-PCV10-type carriage increased by $71 \%$.

Interpretation: Introduction of PCV10 in Kenya resulted in a substantial reduction in PCV10-type IPD in children and adults without significant replacement disease. These findings suggest that routine infant PCV10 immunization programmes with catch-up campaigns will provide substantial direct and indirect protection in low-income settings in tropical Africa.

Funding: Gavi, The Vaccine Alliance; The Wellcome Trust of Great Britain 


\section{Background}

The number of pneumococcal deaths in children aged 1-59 months was estimated at 332,000 globally in 2015, a decline of more than $50 \%$ from $2000 .{ }^{1}$ In middle- and high-income countries, inclusion of pneumococcal conjugate vaccines (PCVs) in routine infant vaccination programmes has led to a substantial reduction in the incidence of invasive pneumococcal disease (IPD) caused by vaccine serotypes (VT). In addition, because vaccinated children are less likely to carry and transmit VT pneumococci, programmatic use of PCV in children has resulted in a decline in IPD in unvaccinated individuals (i.e., "herd protection"). ${ }^{2}$ Evidence of impact of PCVs in low income settings is sparse and impact models rely upon efficacy estimates from randomized controlled trials, not real-world implementation. Furthermore, there is no evidence of the impact of 10-valent PCV conjugated to non-typeable Haemophilus influenzae (PCV10) on invasive pneumococcal disease in Africa, where the greatest burden of deaths from pneumococcal disease occur.

Sixty-one percent (82.4 million) of the world's infants have not received PCV; however, dozens of low-income countries have recently introduced PCV or will do so in the coming decade. ${ }^{3}$ In 2011, with support from Gavi, the Vaccine Alliance, Kenya became one of the first countries in Africa to introduce PCV and the first country to use PCV10. PCV10 was introduced in the Kenyan national childhood immunization schedule as a 3-dose series administered at 6,10 , and 14 weeks of age. There is good evidence of the efficacy of PCV9 in African settings, but efficacy of PCV10 (Synflorix) has not been demonstrated. ${ }^{4,5}$ Furthermore, because of the potential for significant herd protection or for serotype replacement disease, the net population benefit of the PCV programme in these settings can only be estimated through longitudinal IPD surveillance. This information is essential to support realistic cost-effectiveness analyses and sustain the commitment of Ministries of Health (MOH) to the PCV programme as countries transition from Gavi support for vaccines to self-financing. 
Through a collaboration between the Kenya MOH, Gavi, and the KEMRI-Wellcome Trust Research

Programme (KWTRP), we used an existing integrated demographic, clinical, and microbiological surveillance system to conduct a prospectively designed assessment of vaccine impact against nasopharyngeal carriage and IPD in children and adults before and after introduction of PCV10 in the routine infant immunization programme in Kenya.

\section{Methods}

\section{Setting}

This study was conducted at KWTRP among residents of the Kilifi Health and Demographic Surveillance System (KHDSS), a rural community on the Kenyan coast covering an area of $891 \mathrm{~km}^{2}$. A census of the KHDSS in 2000 defined the resident population, and all subsequent births, deaths and migration events were monitored by fieldworker visits to every participating household at approximately 4-monthly intervals. ${ }^{6}$ The population (179568 in 1999; 239392 in 2007; 284826 in 2016) is served by a single government hospital, Kilifi County Hospital $(\mathrm{KCH})$. Among women attending antenatal care at $\mathrm{KCH}$, the prevalence of HIV infection ranged between $2 \cdot 1 \%$ and $4 \cdot 6 \%$ during $2005-2016$ (Supplemental Figure 1 ). The prevalence of HIV among children $<5$ years in Kenya was estimated in 2012 at 1.6\%. ${ }^{7}$ Haemophilus influenzae type b conjugate vaccine was introduced in Kenya in $2001 .^{8}$ In the 12 years prior to PCV10 introduction, the serotypes contained in PCV10 $(1,4,5,6 B, 7 F, 9 V, 14,18 C, 19 F$, and 23F) comprised 75\% of IPD in children aged $<5$ years in Kilifi.

\section{Vaccine introduction and monitoring}

In January 2011, the government of Kenya introduced PCV10 into the national immunization schedule, administered simultaneously with Pentavalent vaccine (diphtheria/whole cell pertussis/tetanus/hepatitis $\mathrm{B} / \mathrm{H}$. influenzae type $\mathrm{b}$ vaccine) at 6,10 and 14 weeks of age. A national catch-up campaign provided three doses of PCV10 to children aged $<12$ months. As part of the study design, the MOH conducted a catch-up 
campaign in Kilifi County providing up to two doses of PCV10 to children aged 12-59 months in two campaigns, beginning on January 31, 2011 and March 21, 2011, each lasting 1-2 weeks. All vaccines were captured by the Kilifi Vaccine Monitoring System, a registry in which data clerks at 26 clinics serving the KHDSS link vaccination at the point of delivery to the child's identification in the KHDSS. ${ }^{9}$ Coverage with PCV10 increased sharply during the catch-up campaign and slowly thereafter (Figure 1, Supplemental Table 1). Coverage with $\geq 2$ PCV10 doses in 2-11 month olds was $80 \%$ in 2011 and $84 \%$ in 2016; coverage with $\geq 1$ dose in $12-59$ month olds was $66 \%$ and $87 \%$, respectively.

\section{Clinical and laboratory procedures}

Children admitted to $\mathrm{KCH}$ (with the exception of trauma patients or patients admitted for elective surgery) were investigated with a blood culture at the time of admission from 1999-2016. ${ }^{10}$ Adults (age $\geq 15$ years) admitted to $\mathrm{KCH}$ from 2007-2016 were investigated with a blood culture at the time of admission if there were signs or symptoms of invasive bacterial disease (e.g., history of fever, axillary temperature $<36.0^{\circ} \mathrm{C}$ or $>37.5^{\circ} \mathrm{C}$, signs of focal sepsis $)^{11}$. Blood was cultured using an automated BACTEC instrument (BD Diagnostics, USA). From 1999-2016, with the exception of a brief change in practice in 2004-2005, the clinical indications for lumbar puncture were impaired consciousness or meningism in children younger than 5 years, prostration in children younger than 3 years, seizures (other than febrile seizures) in children younger than 2 years and suspicion of sepsis in children younger than 60 days, or suspected meningoencephalitis in adults. Cerebrospinal fluid (CSF) was cultured on horse blood and chocolate agar. Admitted patients were tested for HIV using two rapid antibody tests according to the Kenya national policy. ${ }^{12}$ Patients were treated according to Kenyan $\mathrm{MOH}$ or WHO guidelines.

Nasopharyngeal carriage of pneumococci was assessed through cross-sectional surveys conducted on annual samples of approximately 500 KHDSS residents of all ages selected at random from the KHDSS population 
register each year from 2009-2016. The methods are described elsewhere, with the exception that flocked swabs (Copan Diagnostics, USA) replaced rayon swabs in $2016 .^{13}$

Isolates of S. pneumoniae from sterile-site and nasopharyngeal swab cultures were identified by optochin susceptibility; serotyping was performed by latex agglutination and Quellung reaction. If pneumococcal colonies of varying appearance were observed, only those of the dominant colony morphology were serotyped. Serogroup 6 isolates were tested by PCR for confirmation of serotype. Invasive isolates from 1999-2008 underwent repeat confirmatory serotyping by Quellung and multiplex PCR. ${ }^{14}$ Invasive isolates from 2008-2016 underwent real-time confirmatory serotyping by PCR; discordant results were resolved by a second PCR. A case of IPD was defined as isolation of S. pneumoniae from a sterile site culture in an individual admitted to $\mathrm{KCH}$ who was resident in the KHDSS. VT isolates were those belonging to PCV10 serotypes $(1,4$, $5,6 \mathrm{~B}, 7 \mathrm{~F}, 9 \mathrm{~V}, 14,19 \mathrm{C}, 19 \mathrm{~F}$, and 23F). All other serotypes were classified as non-VT. Pneumococcal meningitis was defined as isolation of S. pneumoniae from cerebrospinal fluid (CSF) or isolation of S. pneumoniae from blood, accompanied by a CSF white blood cell count of $\geq 50 \times 10^{6}$ cells/L or a ratio of CSF glucose to plasma glucose of $<0 \cdot 1 .{ }^{15}$ Pneumococcal pneumonia was defined as a case of IPD in a child with cough or difficulty breathing, and $\geq 1$ of the following: lower chest wall indrawing, central cyanosis, inability to drink, convulsions, lethargy, prostration, head nodding. ${ }^{16}$

\section{Statistical analysis}

We designated January 1, 1999 through December 31, 2010, as the pre-vaccine era and January 1, 2012 through December 31, 2016, as the post-vaccine era. The year of vaccine introduction, 2011, was excluded from the analysis of impact. We calculated the age-stratified incidence of IPD in each year as the annual number of cases divided by the mid-year population in the KHDSS. We excluded admissions and person-years of observation during health care worker strikes (Supplemental Table 2). Unadjusted incidence rate ratios 
(IRRs) were calculated for the post-vaccine era compared to the pre-vaccine era by age group using negative binomial regression because of over-dispersion in the data. Possible confounders of the association between IPD and vaccine introduction included time (year), annual incidence of admissions, malaria admissions (i.e., presence of malaria parasites by microscopy), moderate or severe malnutrition admissions (among children $<5$ years; defined as weight-for-age $>-2$ z-scores below the median of the WHO child growth standards), and compliance with recommendations for investigation by blood culture. Potential confounders with a $p$-value $<0.1$ in univariate analysis were included in multivariable analysis; we used backward stepwise regression and excluded variables with a likelihood ratio test $p$-value $\geq 0 \cdot 05$. We built age-group specific models for overall IPD and applied the same structure within age-group for VT and non-VT IPD. The percent reduction in disease was calculated as 1 minus the adjusted IRR.

We calculated pneumococcal carriage prevalence ratios comparing nasopharyngeal carriage in the pre- and post-vaccine eras as previously described. ${ }^{13}$ Briefly, prevalence ratios were modeled using log-binomial regression; if the models failed to converge we used Poisson regression with robust confidence intervals. Adjusted prevalence ratios were age-standardized to reflect the stratified sampling scheme using the inverse of the sampling ratio as population weights.

The significance of vaccine impact on serotype-specific IPD or carriage was tested using a Bonferroni correction (i.e., for 25 serotypes, the correction was $0 \cdot 05 / 25$ ).

STATA $14 \cdot 0$ (Stata Corp, USA) was used for analysis.

\section{Ethical approval}

The protocol was approved by the Oxford Tropical Ethical Review Committee (No. 30-10) and the Kenya National Ethical Review Committee (SSC1433). Adult participants and parents/guardians of all child participants provided written informed consent. 


\section{Role of the funding source}

The study was funded by Gavi, The Vaccine Alliance and The Wellcome Trust. The funders had no role in the study design; in the collection, analysis, and interpretation of data; in the writing of the report; nor in the decision to submit the paper for publication.

\section{Results}

During the 18-year surveillance period, we identified 667 cases of IPD in 3,211,403 person years of observation among KHDSS residents (Supplemental Table 3). The specimen type and proportion of IPD cases with HIV infection did not change significantly between the pre- and post-vaccine eras (Table 1). Among children $<5$ years, the median age of IPD cases was 14 months (interquartile range $[I Q R] 7,30$ ) in the prevaccine era and 20 months (IQR 6, 38) in the PCV10 era. Throughout the 18 -year surveillance period several indicators suggested marked improvements in the overall health of the population (Supplemental Figure 2; Supplemental Figure 3).

Among children $<5$ years, the incidence of VT-IPD declined from $60 \cdot 8$ to $3 \cdot 2 / 100000$ in the pre- and postvaccine eras (Table 2; Figure 2; Supplemental Table 4) representing a reduction of $92 \%$ (95\% Cl: 78, 97; adjusted for year). The average annual number of VT-IPD cases fell from 25 (IQR 16, 33) in the pre-vaccine era to $1(I Q R 1,2)$ in the post-vaccine era. Seven children had VT-IPD in the post-vaccine era: two were unvaccinated and five were age-appropriately vaccinated (Supplemental Table 5). Of the five children who developed VT-IPD after receipt of PCV10, two were noted to have underlying conditions (malnutrition in both). A decline in incidence was observed for all PCV10 serotypes, and this was statistically significant for serotypes 1 and 14, the most common serotypes in the pre-vaccine era. The incidence of non-VT IPD did not significantly increase following introduction of PCV10 (IRR 1.31; 95\% CI: 0.65, 2.64). Overall, use of PCV10 led 
to a $68 \%(95 \% \mathrm{Cl}: 40,83)$ reduction in the incidence of all-serotype IPD, and an $85 \%(95 \% \mathrm{Cl}: 66,93)$

reduction in the incidence of bacteraemic pneumococcal pneumonia among children $<5$ years.

Among children too young to be vaccinated (i.e., $<2$ months of age), the incidence of VT-IPD declined from 173.2 to 0/100000. A significant decline in the rate of VT-IPD was also observed among individuals 5-14 years (74\% reduction, $95 \% \mathrm{Cl}: 41,89$; adjusted for blood culture ascertainment), and $\geq 15$ years ( $81 \%$ reduction, 95\% Cl: 49, 93; adjusted for blood culture ascertainment) (Figure 2; Table 2, Supplemental Table 3). There was no significant change in the incidence of non-VT IPD in these age groups (Table 2).

Overall 4,066 KHDSS residents were enrolled in the nasopharyngeal carriage surveys (Supplemental Table 6). VT carriage declined among individuals $<5$ years (with statistically significant reductions for serotypes $6 \mathrm{~B}, 19 \mathrm{~F}$ and 23F), 5-14 years, and $\geq 15$ years. Non-VT carriage increased significantly in in all age groups (Table 3; Supplemental Figure 4). Among children $<5$ years, carriage of vaccine-related serotype 19A increased; no effect on carriage of serotype 6A was observed. In 2016, carriage of VT pneumococci was detected in 10/156 (6\%) of children $<5$ years, all of whom had received 3 doses of PCV10, and 7/99 (7\%) of children 5-14 years, none of whom had received PCV10.

\section{Discussion}

Using a longstanding, integrated clinical, laboratory and demographic surveillance system, we documented a 92\% reduction in VT-IPD in children aged $<5$ years and substantial indirect protection in Kilifi, Kenya following introduction of PCV10 to the routine infant immunization schedule, accompanied by a catch-up campaign. Kenya was the first African country to include PCV10 in its routine childhood immunization programme. This study provides the first population-level evidence of a robust direct and indirect effect of a PCV10 
programme in a low-income country and does not find significant evidence of serotype replacement disease in the first six years of PCV10 use.

Following introduction of PCVs, a large decline in IPD was documented in numerous developed world settings; however, serotype distributions in carriage and IPD differ by geographic area and there have been few opportunities to examine PCV10 impact in a developing country. ${ }^{17}$ In Kilifi, we observed a $68 \%$ reduction in overall IPD and a 92\% reduction in VT-IPD among children $<5$ years, consistent with findings in middle- and high-income settings using either PCV10 or PCV13. Four years after PCV13 introduction, IPD was reduced by $64 \%$ in US children $<5$ years and $46 \%$ in British children $<2$ years. ${ }^{18,19}$ Within the first $3-5$ years of PCV13 use, the rate of IPD caused by the 6 serotypes present in PCV13 but not PCV7 declined $93 \%$ in US children $<5$ years, $80 \%$ in Alaska Native children $<5$ years, $89 \%$ in British children $<2$ years, and $82 \%$ in Gambian children 2-23 months. ${ }^{18-21}$ In Latin American countries using PCV10 or PCV13, effectiveness against VT-IPD has been estimated at $56 \%$ to $84 \% .{ }^{22}$ Contributing to the observed reduction in IPD in Kilifi were an $85 \%$ reduction in bacteraemic pneumococcal pneumonia incidence and a $69 \%$ reduction in pneumococcal meningitis incidence in children $<5$ years. PCV impact on these important clinical outcomes has been documented elsewhere. ${ }^{23-25}$ Given that the majority of pneumococcal disease is comprised of pneumonia, it is notable that PCV10 introduction in Kenya was associated with reductions in childhood hospitalisations with clinically-defined and radiologically-confirmed pneumonia of $27 \%$ and $48 \%$, respectively. ${ }^{26}$

In the pre-vaccine era, IPD was driven by epidemics of serotypes 1 and 5 in Kilifi. PCV10 use not only reduced the incidence of disease but obliterated IPD epidemics, as was also observed in the US. ${ }^{27}$ We did not observe a reduction in IPD caused by vaccine-related serotypes 6A or 19A; this is consistent with the findings from the nasopharyngeal carriage surveys in Kilifi. In contrast, a recent analysis of the long-term impact of PCV10 in Finland documented a reduction in IPD caused by serotype 6A (but not $19 \mathrm{~A}$ ). ${ }^{28}$ 
Notably, we observed protection among infants too young to be vaccinated and among older children and adults. This contrasts with findings from The Gambia, where indirect effects were not observed. ${ }^{21} \mathrm{~A}$ catch-up campaign was not undertaken in The Gambia but in Kilifi this likely accelerated population protection. ${ }^{29}$ In South Africa a decline in VT-IPD was noted among adults aged 25-44 years within 3 years of PCV7 introduction. $^{30}$

The indirect protection afforded by PCVs is driven by the reduction in nasopharyngeal carriage of VT pneumococci among vaccinated children. In Kilifi, there was a significant reduction in carriage of VT pneumococci in both vaccinated and unvaccinated populations within 6 months of PCV10 introduction. ${ }^{13}$ However, while vaccine-type carriage has declined, VT pneumococci continue to be identified in $6 \%$ of children $<5$ years and $8 \%$ of infants in Kilifi, compared to $<1-2 \%$ in other countries that use PCVs. ${ }^{31-35}$ This may reflect a higher force of infection in Kilifi and it indicates continued risk for VT-IPD in unvaccinated or undervaccinated children, and adults. ${ }^{36}$ An alternative explanation for the persistence of VT carriage in Kilifi is that, unlike most middle-income and high-income countries, Kenya introduced PCV10 without a booster dose in the second year of life. Many low-income countries have introduced PCV with three primary doses without a booster ( $3+0$ schedule), and it will be important to determine whether the absence of a booster dose, which may be accompanied by waning immunity, leads to a persistent transmission reservoir, vaccination failures or rebound disease incidence.

In addition to persistent VT carriage, we also observed a 71\% increase in carriage of non-VT pneumococci (particularly serotype 19A) in children $<5$ years. The PCV-associated decline in VT carriage and corresponding increase in non-VT carriage has been well-described. Although an increase in non-VT IPD disease has been reported in among some age groups in several settings that use expanded valency PCVs, the increases have 
been small compared to the decline in VT-IPD. ${ }^{18,19}$ The only exception to this is a recent increase in total IPD incidence among persons 5-64 and $\geq 65$ years of age in North East England that was observed seven years following introduction of PCV13 in the routine vaccination program in the United Kingdom. ${ }^{37}$ Although our surveillance for non-VT IPD did not detect a significant increase in any age group, the direction of change was positive in all age-groups $\geq 2$ months (IRRs 1·31-1·47). Given the low baseline incidence of non-VT IPD, comprising only one quarter of the pre-vaccine burden of IPD among children $<5$ years, the power of the study was only sufficient (i.e., $>80 \%$ ) to detect a $\geq 2 \cdot 3$-fold change (Supplemental Table 7). The small relative increase that was observed did not translate into a significant absolute rise in incidence. To clarify these emerging trends, it will be important to continue to monitor children and adults for pneumococcal disease in the present surveillance setting for several years to come.

The before-after study design, which is the principal method for evaluation of population impact of vaccines, has inherent weaknesses. In Kilifi, general health improved slowly over the surveillance period. The incidence of hospital admissions for a variety of illnesses declined. The reduction in non-VT IPD over time among infants $<2$ months, in contrast to the rise in older age groups, suggests specific improvements in maternity services and infant care. In an exploratory post-hoc analysis using negative binomial regression, adjusting for calendar-year, the introduction of PCV10 was associated with a non-statistically significant $35 \%$ reduction in invasive $S$. aureus disease in children $<5$ years (IRR $0.65 ; 95 \% \mathrm{CI}: 0 \cdot 36,1 \cdot 18)$. The incidence of HIV in the population has not been systematically measured and was not included in the analysis; however, the prevalence of HIV among women seeking ante-natal care was $<5 \%$ over the surveillance period and it is therefore unlikely that changes in HIV incidence would have significantly confounded estimates of vaccine effectiveness. Overall, several factors argue that the observed reduction in IPD in Kilifi is attributable to the introduction of PCV10. Consistent surveillance methods were used over a long period of time and changes in VT-IPD occurred abruptly at the same time as vaccine introduction with a catch-up campaign, and 
simultaneously with marked changes in VT carriage prevalence. Systematically collected data on a wide range of possible confounders were included in the analysis.

Pneumococcal disease remains a leading vaccine-preventable cause of childhood mortality, and the majority of these deaths occur in Africa. To date, 141 countries, including 58 Gavi-eligible countries, have introduced a PCV into their national childhood immunization programmes. ${ }^{3}$ Currently, sixteen countries were in the process of transitioning out of Gavi support while five had reached the end of Gavi support. The PCV programme is the most expensive component of the national immunization schedule and the sustainability of PCV vaccination in low-income countries will depend on the demonstrable impact of PCV in reducing childhood morbidity and mortality. Because of the necessity for stable pre-vaccine surveillance, evaluations of PCV impact are rare in Africa. Based on this carefully standardized, prospectively designed, 18-year surveillance study, we conclude that use of PCV10 in tropical Africa will lead to substantial and sustained health benefits for the whole population. 


\section{Declaration of interests}

LLH has received research funding outside this work through her institution from Novavax, GlaxoSmithKline Biologicals, Merck, and Pfizer, Inc. JCM is currently employed by Pfizer. All other authors declare no conflicts of interest.

Funding: Gavi, The Vaccine Alliance; AOE, KM, TNW and JAGS were funded by the Wellcome Trust

(Fellowship numbers: 103951, 203077, 202800 and 098532, respectively). The funding sources had no role in the study design; collection, analysis and interpretation of data; and in the decision to submit for publication.

\section{Acknowledgements}

We thank the residents of the KHDSS, the Ministry of Health Sub-County Health Management Team in Kilifi County, and the dedicated team of field workers, administrative staff, clinicians and laboratorians who worked on this study. This paper is published with the permission of the Director, Kenya Medical Research Institute. 


\section{References}

1. Wahl B, O'Brien KL, Greenbaum A, et al. Global burden of Streptococcus pneumoniae in children younger than 5 years in the era of pneumococcal conjugate vaccines (PCV): 2000-2015. 10th International Symposium on Pneumococci and Pneumococcal Disease, Glasgow, Scotland 2016.

2. Tsaban G, Ben-Shimol S. Indirect (herd) protection, following pneumococcal conjugated vaccines introduction: A systematic review of the literature. Vaccine 2017; 35(22): 2882-91.

3. IVAC. VIEW-hub. 2018. http://view-hub.org/ (accessed January 25, 2018).

4. Cutts FT, Zaman SM, Enwere G, et al. Efficacy of nine-valent pneumococcal conjugate vaccine against pneumonia and invasive pneumococcal disease in The Gambia: randomised, double-blind, placebo-controlled trial. Lancet 2005; 365(9465): 1139-46.

5. Klugman KP, Madhi SA, Huebner RE, Kohberger R, Mbelle N, Pierce N. A trial of a 9-valent pneumococcal conjugate vaccine in children with and those without HIV infection. N Engl J Med 2003; 349(14): 1341-8.

6. Scott JA, Bauni E, Moïsi JC, et al. Profile: The Kilifi Health and Demographic Surveillance System (KHDSS). Int J Epidemiol 2012; 41(3): 650-7.

7. National AIDS and STI Control Program (NASCOP) K. Kenya AIDS Indicator Survey 2012: Final Report. Nairobi, 2014.

8. Cowgill KD, Ndiritu M, Nyiro J, et al. Effectiveness of Haemophilus influenzae type b conjugate vaccine introduction into routine childhood immunization in Kenya. JAMA 2006; 296(6): 671-8.

9. Adetifa IMO, Bwanaali T, Wafula J, et al. Cohort Profile: The Kilifi Vaccine Monitoring Study. Int J Epidemiol 2017; 46(3): 792-h.

10. Berkley JA, Lowe BS, Mwangi I, et al. Bacteremia among children admitted to a rural hospital in Kenya. N Engl J Med 2005; 352(1): 39-47.

11. Etyang AO, Munge K, Bunyasi EW, et al. Burden of disease in adults admitted to hospital in a rural region of coastal Kenya: an analysis of data from linked clinical and demographic surveillance systems. Lancet Glob Health 2014; 2(4): e216-24.

12. Clnical Management and Referral Guidelines. Nairobi, Kenya: Kenya Ministry of Medical Services and Ministry of Public Health and Sanitation; 2009.

13. Hammitt LL, Akech DO, Morpeth SC, et al. Population effect of 10 -valent pneumococcal conjugate vaccine on nasopharyngeal carriage of Streptococcus pneumoniae and non-typeable Haemophilus influenzae in Kilifi, Kenya: findings from cross-sectional carriage studies. Lancet Glob Health 2014; 2(7): e397-405. 
14. Pai R, Gertz RE, Beall B. Sequential multiplex PCR approach for determining capsular serotypes of Streptococcus pneumoniae isolates. J Clin Microbiol 2006; 44(1): 124-31.

15. Berkley JA, Mwangi I, Ngetsa CJ, et al. Diagnosis of acute bacterial meningitis in children at a district hospital in sub-Saharan Africa. Lancet 2001; 357(9270): 1753-7.

16. WHO. Pocket book of hospital care for children. Guidelines for the management of common illnesses with limited resources 2005.

17. Johnson HL, Deloria-Knoll M, Levine OS, et al. Systematic evaluation of serotypes causing invasive pneumococcal disease among children under five: the pneumococcal global serotype project. PLoS Med 2010; 7(10).

18. Moore MR, Link-Gelles R, Schaffner W, et al. Effect of use of 13-valent pneumococcal conjugate vaccine in children on invasive pneumococcal disease in children and adults in the USA: analysis of multisite, population-based surveillance. Lancet Infect Dis 2015; 15(3): 301-9.

19. Waight PA, Andrews NJ, Ladhani SN, Sheppard CL, Slack MP, Miller E. Effect of the 13-valent pneumococcal conjugate vaccine on invasive pneumococcal disease in England and Wales 4 years after its introduction: an observational cohort study. Lancet Infect Dis 2015; 15(5): 535-43.

20. Bruce MG, Singleton R, Bulkow L, et al. Impact of the 13-valent pneumococcal conjugate vaccine (PCV13) on invasive pneumococcal disease and carriage in Alaska. Vaccine 2015; 33(38): 4813-9.

21. Mackenzie GA, Hill PC, Jeffries DJ, et al. Effect of the introduction of pneumococcal conjugate vaccination on invasive pneumococcal disease in The Gambia: a population-based surveillance study. Lancet Infect Dis 2016; 16(6): 703-11.

22. de Oliveira LH, Camacho LA, Coutinho ES, et al. Impact and effectiveness of 10 and 13-valent pneumococcal conjugate vaccines on hospitalization and mortality in children aged less than 5 years in Latin American countries: a systematic review. PLOS ONE 2016; 11(12): e0166736.

23. Ben-Shimol S, Givon-Lavi N, Grisaru-Soen G, et al. Comparative incidence dynamics and serotypes of meningitis, bacteremic pneumonia and other-IPD in young children in the PCV era: Insights from Israeli surveillance studies. Vaccine 2017.

24. Hsu HE, Shutt KA, Moore MR, et al. Effect of pneumococcal conjugate vaccine on pneumococcal meningitis. N Engl J Med 2009; 360(3): 244-56.

25. Nhantumbo AA, Weldegebriel G, Katsande R, et al. Surveillance of impact of PCV-10 vaccine on pneumococcal meningitis in Mozambique, 2013 - 2015. PLoS One 2017; 12(6): e0177746.

26. S Silaba M, Ooko M, Bottomley C, et al. The impact of 10 -valent Pneumococcal Conjugate Vaccine on the incidence of radiologically-confirmed pneumonia and on pneumonia hospitalizations among children in 
Kilifi, Kenya. 10th International Symposium on Pneumococci and Pneumococcal Diseases. Glasgow, Scotland 2016.

27. Walter ND, Taylor TH, Jr., Dowell SF, Mathis S, Moore MR. Holiday spikes in pneumococcal disease among older adults. N Engl J Med 2009; 361(26): 2584-5.

28. Rinta-Kokko H, Palmu AA, Auranen K, et al. Long-term impact of 10-valent pneumococcal conjugate vaccination on invasive pneumococcal disease among children in Finland. Vaccine 2018; 36(15): 1934-40.

29. Flasche S, Ojal J, Le Polain de Waroux O, et al. Assessing the efficiency of catch-up campaigns for the introduction of pneumococcal conjugate vaccine: a modelling study based on data from PCV10 introduction in Kilifi, Kenya. BMC Med 2017; 15(1): 113.

30. von Gottberg A, de Gouveia L, Tempia S, et al. Effects of vaccination on invasive pneumococcal disease in South Africa. N Engl J Med 2014; 371(20): 1889-99.

31. Desai AP, Sharma D, Crispell EK, et al. Decline in pneumococcal nasopharyngeal carriage of vaccine serotypes after the introduction of the 13-valent pneumococcal conjugate vaccine in children in Atlanta, Georgia. Pediatr Infect Dis J 2015; 34(11): 1168-74.

32. Dunais $B$, Bruno $P$, Touboul $P$, et al. Impact of the 13-valent pneumococcal conjugate vaccine on nasopharyngeal carriage of Streptococcus pneumoniae among children attending group daycare in southeastern France. Pediatr Infect Dis J 2015; 34(3): 286-8.

33. Grant LR, Hammitt LL, O'Brien SE, et al. Impact of the 13-valent pneumococcal conjugate vaccine on pneumococcal carriage among American Indians. Pediatr Infect Dis J 2016; 35(8): 907-14.

34. van Hoek AJ, Sheppard CL, Andrews NJ, et al. Pneumococcal carriage in children and adults two years after introduction of the thirteen valent pneumococcal conjugate vaccine in England. Vaccine 2014; 32(34): 4349-55.

35. Nzenze SA, Shiri T, Nunes MC, et al. Temporal changes in pneumococcal colonization in a rural African community with high HIV prevalence following routine infant pneumococcal immunization. The Pediatric infectious disease journal 2013; 32(11): 1270-8.

36. Choi YH MA, van Hoek AJ, Roca A, Mackenzie G, Gay N. Impact of thirteen-valent pneumococcal conjugate vaccine on pneumococcal carriage in different countries - mathematical modelling study. 9th International Symposium on Pneumococci and Pneumococcal Diseases. Hyderabad, India; 2014.

37. Houseman C, Hughes GJ, Chapman KE, Wilson D, Gorton R. Increased invasive pneumococcal disease, North East England, UK. Emerg Infect Dis 2017; 23(1): 122-6. 
Table 1. Invasive pneumococcal disease among children and adults in the KHDSS admitted to the Kilifi County Hospital, in the pre-vaccine (1999-2010) and post-vaccine (2012-2016) eras.

\section{Pre-vaccine era \\ (1999-2010)}

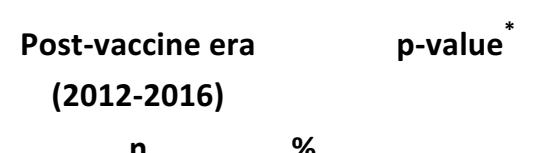$$
\text { Ase }
$$

Age $<5$ years

Total admissions

Mean annual admissions

Recommended for blood culture

Blood culture collected $\dagger$

Culture-confirmed IPD

Age $<24$ months

Male

Cultured from blood specimen

Cultured from CSF

Pneumococcal meningitisł

HIV-infected

Died during the episode
Age 5-14 Years
Total admissions
Mean annual admissions
Recommended for blood culture
Blood culture collected $†$
Culture-confirmed IPD

Male

Cultured from blood specimen

Cultured from CSF

Pneumococcal meningitisł

HIV-infected

Died during the episode

n $\quad \%$

31,422

2,618

30,098

29,234

401

273

229

390

71

78

25

70

5,296

441

4,483

4,200

127

66

121

31

37

7

30
$\%$

96
97
68
57
97
18
62
20
17

17

(2)

\section{6,323}

1,264

\section{5,691}

5,353

34

20

23

34

3

3

3
4

11

\section{2,060}

412

1,592

1,438

85

94

22

$52 \quad 10$

10

95

24

76

23

24

$\begin{array}{rr}5,332 & \\ 1,326 & \\ 5,048 & 95 \\ 2,107 & 42 \\ 30 & \\ 12 & 40 \\ 29 & 97 \\ 4 & 33 \\ 12 & 40\end{array}$

\section{5,163}

1,032

$0 \cdot 10$

Mean annual admissions

Recommended for blood culture

Blood culture collected $\dagger$

4,597

1,875

26

26

11

23

14

12

Culture-confirmed IPD

Cultured from blood specimen

HIV-infected

Died during the episode

$\%$

\footnotetext{
Abbreviations: IPD, invasive pneumococcal disease; CSF, cerebrospinal fluid

*p-value computed by t-test for comparison of means and chi-square for comparison of percentages.

†Among those recommended for blood culture

$\ddagger$ Defined as isolation of $S$. pneumoniae from CSF or isolation of S. pneumoniae from blood, accompanied by a CSF white blood cell count of $50 \times 10^{6}$ cells/L or greater or a ratio of CSF glucose to plasma glucose less than $0 \cdot 1 .{ }^{15}$
} 
Table 2. Incidence (per 100,000) of invasive pneumococcal disease among children and adults in the KHDSS, in the pre- and post-vaccine eras.

\begin{tabular}{|c|c|c|c|c|c|c|c|c|c|c|}
\hline \multirow{2}{*}{$\begin{array}{c}\text { Serotype group } \\
\text { and age } \\
\text { category }\end{array}$} & \multicolumn{3}{|c|}{ Pre-vaccine era (1999-2010) } & \multicolumn{3}{|c|}{ Post-vaccine era (2012-2016) } & \multicolumn{4}{|c|}{ Post- vs pre-vaccine era } \\
\hline & $\mathbf{n}$ & $\begin{array}{c}\text { Incidence } \\
\text { per } 100,000\end{array}$ & $95 \% \mathrm{Cl}$ & $\mathbf{n}$ & $\begin{array}{c}\text { Incidence } \\
\text { per } 100,000\end{array}$ & $95 \% \mathrm{Cl}$ & IRR' & $95 \% \mathrm{Cl}$ & Adjusted IRR $\ddagger$ & $95 \% \mathrm{Cl}$ \\
\hline \multicolumn{11}{|l|}{ All-type IPD } \\
\hline$<2$ months & 43 & $240 \cdot 2$ & $173 \cdot 9,323 \cdot 6$ & 1 & $7 \cdot 9$ & $0 \cdot 2,44 \cdot 0$ & 0.03 & $0.00,0.25$ & $0 \cdot 13$ & $0 \cdot 01,1 \cdot 24$ \\
\hline$<5$ years & 401 & $81 \cdot 6$ & $73 \cdot 8,89 \cdot 9$ & 34 & $15 \cdot 3$ & $10 \cdot 6,21 \cdot 4$ & $0 \cdot 18$ & $0.11,0.29$ & $0 \cdot 32$ & $0.17,0.60$ \\
\hline $5-14$ years & 127 & $15 \cdot 8$ & $13 \cdot 2,18 \cdot 8$ & 22 & $5 \cdot 5$ & $3 \cdot 5,8 \cdot 4$ & $0 \cdot 34$ & $0.17,0.67$ & 0.47 & $0.24,0.92$ \\
\hline$\geq 15$ years & 30 & $2 \cdot 4$ & $1 \cdot 6,3 \cdot 4$ & 26 & $3 \cdot 4$ & $2 \cdot 5,5 \cdot 7$ & 0.56 & $0.33,0.96$ & 0.63 & $0.36,1.08$ \\
\hline \multicolumn{11}{|l|}{ PCV10-type } \\
\hline$<2$ months & 31 & $173 \cdot 2$ & $117 \cdot 7,245 \cdot 8$ & 0 & $0 \cdot 0$ & & Not estimable & & & \\
\hline$<5$ years & 299 & $60 \cdot 8$ & $54 \cdot 1,68 \cdot 1$ & 7 & $3 \cdot 2$ & $1 \cdot 3,6 \cdot 5$ & 0.05 & $0.02,0.12$ & 0.08 & $0.03,0.22$ \\
\hline $5-14$ years & 105 & $13 \cdot 1$ & $10 \cdot 7,15 \cdot 8$ & 10 & $2 \cdot 5$ & $1 \cdot 2,4 \cdot 6$ & $0 \cdot 19$ & $0.08,0.43$ & $0 \cdot 26$ & $0.11,0.59$ \\
\hline$\geq 15$ years & 20 & $1 \cdot 6$ & $1 \cdot 0,2 \cdot 4$ & 5 & $0 \cdot 8$ & $0 \cdot 2,1 \cdot 7$ & $0 \cdot 16$ & $0.06,0.45$ & $0 \cdot 19$ & $0.07,0.51$ \\
\hline \multicolumn{11}{|l|}{ Non-PCV10 type } \\
\hline$<2$ months & 12 & $67 \cdot 0$ & $34 \cdot 3,117 \cdot 0$ & 1 & $7 \cdot 9$ & $0 \cdot 2,4 \cdot 0$ & $0 \cdot 12$ & $0.02,0.94$ & $0 \cdot 26$ & $0 \cdot 02,3 \cdot 45$ \\
\hline$<5$ years & 102 & $20 \cdot 8$ & $16 \cdot 9,25 \cdot 2$ & 27 & $12 \cdot 2$ & $8 \cdot 0,17 \cdot 7$ & $0 \cdot 58$ & $0.35,0.95$ & $1 \cdot 31$ & $0 \cdot 65,2 \cdot 64$ \\
\hline $5-14$ years & 22 & $2 \cdot 7$ & $1 \cdot 7,4 \cdot 2$ & 12 & $3 \cdot 0$ & $1 \cdot 6,5 \cdot 3$ & $1 \cdot 10$ & $0 \cdot 54,2 \cdot 22$ & $1 \cdot 45$ & $0 \cdot 66,3 \cdot 20$ \\
\hline$\geq 15$ years & 10 & $0 \cdot 8$ & $0.4,1 \cdot 5$ & 21 & $3 \cdot 1$ & $1 \cdot 9,4 \cdot 8$ & $1 \cdot 36$ & $0.62,2.98$ & $1 \cdot 47$ & $0 \cdot 67,3 \cdot 21$ \\
\hline \multicolumn{11}{|c|}{ All-type pneumococcal pneumonia } \\
\hline$<5$ years & 212 & $43 \cdot 1$ & $37 \cdot 5,49 \cdot 3$ & 19 & $8 \cdot 6$ & $5 \cdot 2,13 \cdot 4$ & $0 \cdot 20$ & $0 \cdot 11,0.35$ & $0 \cdot 15$ & $0.07,0.34$ \\
\hline $5-14$ years & 57 & $7 \cdot 1$ & $5 \cdot 4,9 \cdot 2$ & 11 & $2 \cdot 8$ & $1 \cdot 4,5 \cdot 0$ & $0 \cdot 38$ & $0 \cdot 17,0.86$ & 0.49 & $0 \cdot 21,1 \cdot 11$ \\
\hline \multicolumn{11}{|c|}{ All-type pneumococcal meningitis } \\
\hline$<5$ years & 78 & $15 \cdot 9$ & $12 \cdot 5,19 \cdot 8$ & 3 & 1.4 & $0 \cdot 3,4 \cdot 0$ & 0.08 & $0.02,0.29$ & $0 \cdot 31$ & $0 \cdot 08,1 \cdot 21$ \\
\hline $5-14$ years & 37 & $4 \cdot 6$ & $3 \cdot 2,6 \cdot 4$ & 4 & $1 \cdot 0$ & $0 \cdot 3,2 \cdot 6$ & $0 \cdot 21$ & $0.06,0.71$ & $0 \cdot 31$ & $0.09,1.00$ \\
\hline
\end{tabular}

Abbreviations: $\mathrm{Cl}$, confidence interval; IRR, incidence rate ratio

*For individuals $\geq 15$ years, the pre-vaccine era was 2007-2010.

†IRR estimated using negative binomial regression.

¥IRR estimated using negative binomial regression, adjusted for confounding factors significant in the age-specific all-type IPD models: year (age groups <2

months and $<5$ years), blood culture collection (age groups $5-14$ years and $\geq 15$ years). 
Table 3. Carriage prevalence and prevalence ratios for nasopharyngeal carriage of Streptococcus pneumoniae and non-typeable Haemophilus influenzae in the pre- and post-vaccine eras.

\begin{tabular}{|c|c|c|c|c|c|c|c|c|}
\hline & \multicolumn{2}{|c|}{$\begin{array}{r}\text { Carriage prevalence } \\
\text { Pre-vaccine era } \\
(2009-2010)\end{array}$} & \multicolumn{2}{|c|}{$\begin{array}{r}\text { Carriage prevalence } \\
\text { Post-vaccine era } \\
(2012-2016)\end{array}$} & \multicolumn{2}{|c|}{$\begin{array}{r}\text { Crude prevalence } \\
\text { ratio (PR) }\end{array}$} & \multicolumn{2}{|c|}{$\begin{array}{r}\text { Age-standardized } \\
\text { adjusted prevalence } \\
\text { ratio (aPR) }\end{array}$} \\
\hline & $\mathrm{n}$ & $\%$ & $\mathbf{n}$ & $\%$ & PR & $95 \% \mathrm{Cl}$ & aPR & $95 \% \mathrm{Cl}$ \\
\hline \multicolumn{9}{|c|}{ All S. pneumoniae } \\
\hline$<5$ years & 229 & $74 \cdot 4$ & 606 & $76 \cdot 0$ & 1.02 & $0 \cdot 95,1 \cdot 10$ & 1.00 & $0.92,1.08$ \\
\hline 5-14 years & 103 & $52 \cdot 6$ & 237 & $48 \cdot 5$ & 0.92 & $0.78,1.08$ & 0.96 & $0 \cdot 81,1 \cdot 13$ \\
\hline$\geq 15$ years & 123 & $24 \cdot 0$ & 287 & $22 \cdot 8$ & 0.95 & $0 \cdot 79,1 \cdot 14$ & 1.00 & $0 \cdot 81,1 \cdot 22$ \\
\hline \multicolumn{9}{|c|}{ Vaccine-type S. pneumoniae } \\
\hline$<5$ years & 104 & $33 \cdot 8$ & 70 & $8 \cdot 8$ & $0 \cdot 26$ & $0 \cdot 20,0 \cdot 34$ & 0.26 & $0 \cdot 19,0 \cdot 35$ \\
\hline $5-14$ years & 30 & $15 \cdot 3$ & 29 & 5.9 & 0.39 & $0.24,0.63$ & $0 \cdot 38$ & $0.22,0.64$ \\
\hline$\geq 15$ years & 29 & $5 \cdot 7$ & 17 & $1 \cdot 4$ & $0 \cdot 24$ & $0.13,0.43$ & 0.23 & $0.12,0.44$ \\
\hline \multicolumn{9}{|c|}{ Non vaccine-type S. pneumoniae } \\
\hline$<5$ years & 125 & $40 \cdot 6$ & 557 & $69 \cdot 9$ & $1 \cdot 72$ & $1.49,1.99$ & $1 \cdot 71$ & $1.47,1.99$ \\
\hline $5-14$ years & 73 & $37 \cdot 2$ & 216 & $44 \cdot 2$ & $1 \cdot 19$ & $0.96,1.46$ & $1 \cdot 25$ & $1 \cdot 01,1 \cdot 54$ \\
\hline$\geq 15$ years & 94 & $18 \cdot 3$ & 286 & $22 \cdot 7$ & $1 \cdot 24$ & $1 \cdot 01,1 \cdot 53$ & $1 \cdot 30$ & $1.05,1.63$ \\
\hline
\end{tabular}

Abbreviations: $\mathrm{Cl}$, confidence interval

"Models adjusted for number of children aged <10 years in household, month of swab collection, cough or rhinorrhea in preceding 14 days (all age groups); antibiotic use in the preceding 14 days also remained in the model for children age 5-14 years. 
Figure 1. Proportion of children vaccinated with PCV10 (A: 0-11 months; B: $12-23$ months; C: $24-59$ months; C: 5-9 years) in the Kilifi Health and Demographic Surveillance System, 2011-2016.
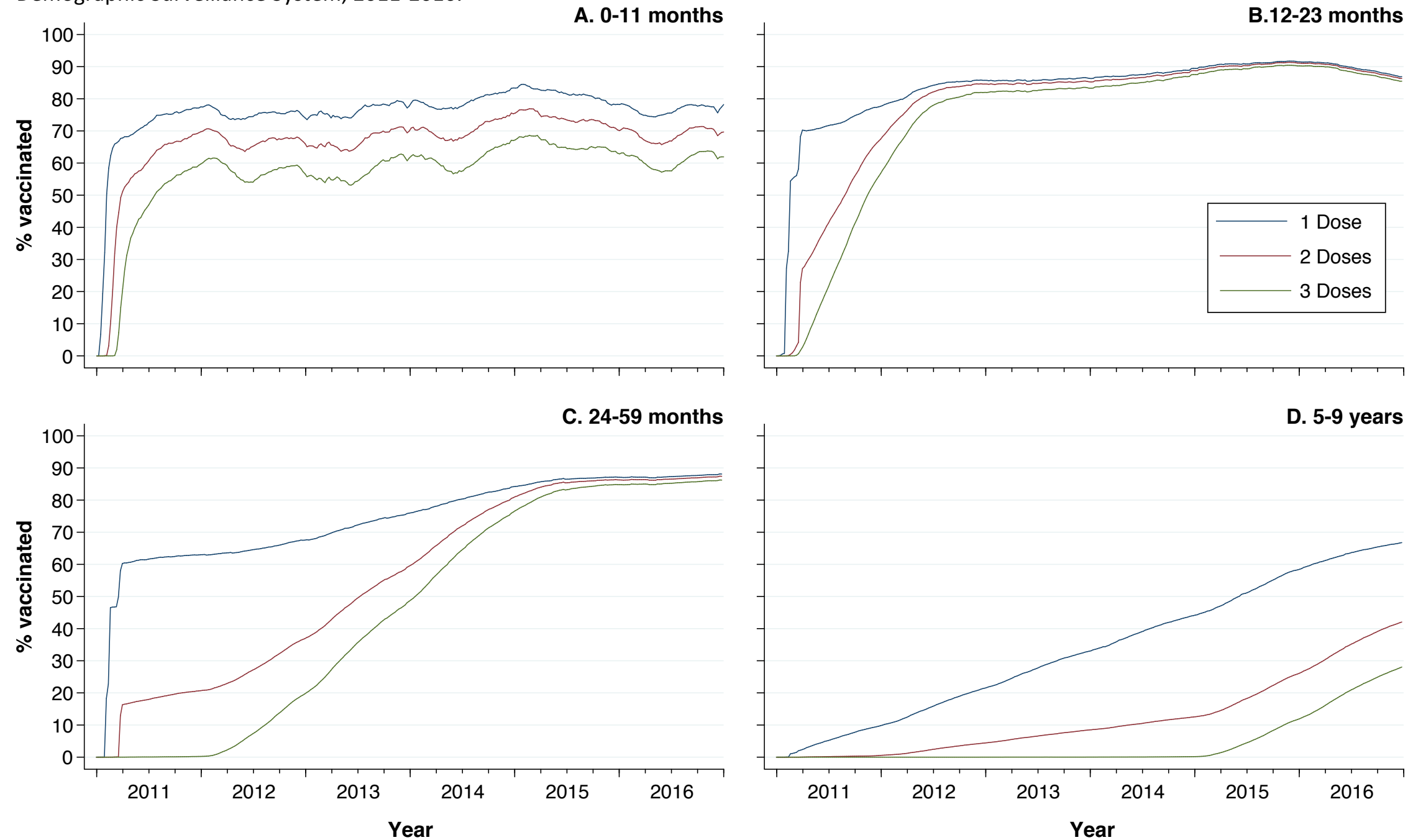
Figure 2. Incidence of overall (black line), vaccine-type (red line) and non-vaccine type (blue line) invasive pneumococcal disease (IPD) in individuals aged A) $<5$ years; B) 5 - 14 years; C) $\geq 15$ years in the Kilifi Health and Demographic Surveillance System, 1999-2016. Vertical dashed line indicates PCV10 introduction.
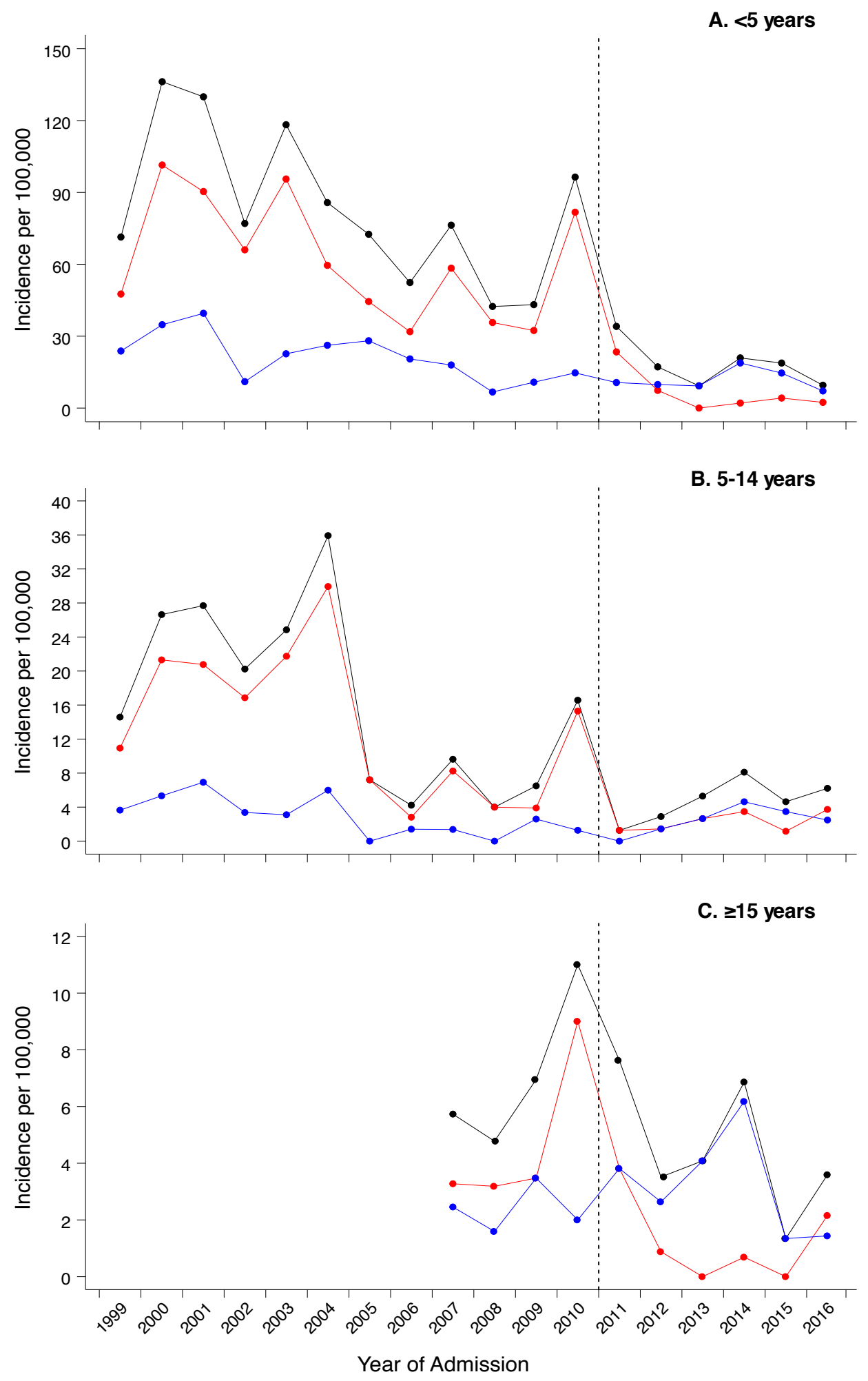

$\longrightarrow$ All IPD $\longrightarrow$ VT-IPD $\longrightarrow$ NVT-IPD 